\title{
A Novel Approach to Selecting Contractor in Agent-based Multi-sensor Battlefield Reconnaissance Simulation
}

\author{
Xiong $\mathbf{L i}{ }^{*}$ \\ Department of Command and Administration, Academy of Armored Force Engineering \\ Beijing, China \\ E-mail: lixiong2609@126.com \\ Zhiming Dong \\ Faculty of Science Research, Academy of Armored Force Engineering \\ Beijing, China \\ E-mail: keny1003@163.com \\ Hao Wang, Qingchao Meng, Zhentian Wang \\ Bengbu Tank Institute \\ Bengbu, Anhui, China \\ E-mail: \{bbtkxywh,bbtkxymqc,bbtkxywzt\}@126.com \\ Yiwei Zhang \\ Graduate School of Engineering, University of Tokyo \\ Tokyo, Japan \\ E-mail: zgyjwczq@126.com \\ Received 9 August 2011 \\ Accepted 27 July 2012
}

\begin{abstract}
This paper presents a novel approach towards showing how contractor in agent-based simulation for complex warfare system such as multi-sensor battlefield reconnaissance system can be selected in Contract Net Protocol (CNP) with high efficiency. We first analyze agent and agent-based simulation framework, CNP and collaborators, and present agents interaction chain used to actualize CNP and establish agents trust network. We then obtain contractor's importance weight and dynamic trust by presenting fuzzy similarity-based algorithm and trust modifying algorithm, thus we propose contractor selecting approach based on maximum dynamic integrative trust. We validate the feasibility and capability of this approach by implementing simulation, analyzing compared results and checking the model.
\end{abstract}

Keywords: agent; agent-based simulation; Contract Net Protocol (CNP); contractor; battlefield reconnaissance system

\section{Introduction}

Agent is an encapsulated computational software system, which is capable of perceiving events in its environment and acting in its environment guided by perceptions and stored information [1-8]. A multi-agent system, as a collection of agents that work together in order to meet an in-community-shared goal [9-13], is designed to cope with a complex problem involving either distributed data, knowledge, or control by a certain interaction

${ }^{*}$ Corresponding Author: Xiong Li

E-mail: lixiong2609@126.com 
mechanism. Agent-based simulation [14-23], as a useful tool to explore complex intelligent systems, is usually applied to describe real entities operation process by representing agents interaction behaviors.

The analysis and comprehensive understanding of complex warfare system is extremely difficult and in most cases impossible for humans to grasp without the assistance of advanced tools such as agent-based simulations, since modern warfare system is complex intelligent system and the demand on quality and costeffectiveness of simulation is rising. On information battlefield, multi-sensor battlefield reconnaissance system (BRS) has heterogeneous members, such as photo-reconnaissance vehicles, electronic reconnaissance vehicles, armored reconnaissance vehicles, unmanned aerial vehicles (UAVs) and information processing vehicles, which have lives and administrative levels. These members have believes, desires, intentions, and it may adopt a role or have relationships with each other. Therefore, it may be seen as a collection of heterogeneous, computational entity agents, i.e., a battlefield reconnaissance multi-agent system, having their own problem solving capabilities and which are able to interact among them in order to reach the overall goal of intelligence reconnaissance.

In order to achieve an agreement among the agents concerning a mutually advantageous cooperation, an effectual collaboration mechanism must be used. Agents interaction protocol has attracted researchers in a surprisingly wide variety of disciplines ranging from computer science and engineering to the military sciences.

In the agent domain, Contract Net Protocol (CNP) has been standardized by the Foundations of Intelligent Physical Agents (FIPA) [24]. CNP [24-30] is the most widely used cooperation and coordination method, and is one of the most popular agents interaction protocols used. CNP is a high level protocol for achieving efficient cooperation based on a market-like protocol. Since BRS acts as a multi-sensor battlefield reconnaissance multi-agent system when agent-based modeling and simulation technology is used to give concept developers a view to the future by enabling them to simulate and manipulate in near real-time operational conditions, it is reasonable to apply CNP to describe and analyze the multi-sensor reconnaissance entity agents interactions.
Thus, a problem is put forward spontaneously: how to select a reconnaissance agent contractor.

With a view to the problem, a lot of research results centre on approaches to selecting rational contractor according to real conditions. However, current results meet a challenge because of many complexities and subtleties in these such as:

- Implementing agent-based simulation through CNP, i.e., enabling communication and interaction among the agents by selecting contractor with its importance weight and dynamic trust.

- Computing the importance weight of a contractor during interactions.

- Modifying the trust of a contractor according to its performance in the former interaction stage.

In this paper, according to the requirement of describing of military agents interactions, the FIPA CNP is extensively used for inter-agent collaboration in dynamic military reconnaissance task scheduling during agent-based multi-sensor battlefield reconnaissance modeling and simulation. To resolve the above problem, we try to design a novel approach to selecting a reconnaissance agent contractor that can combine information of its fuzzy similarity-based importance weight and dynamic trust for reliable battlefield reconnaissance actions simulation.

\section{Agent-based Multi-sensor Battlefield Reconnaissance Simulation}

\subsection{Agent and Agent-based Simulation Framework}

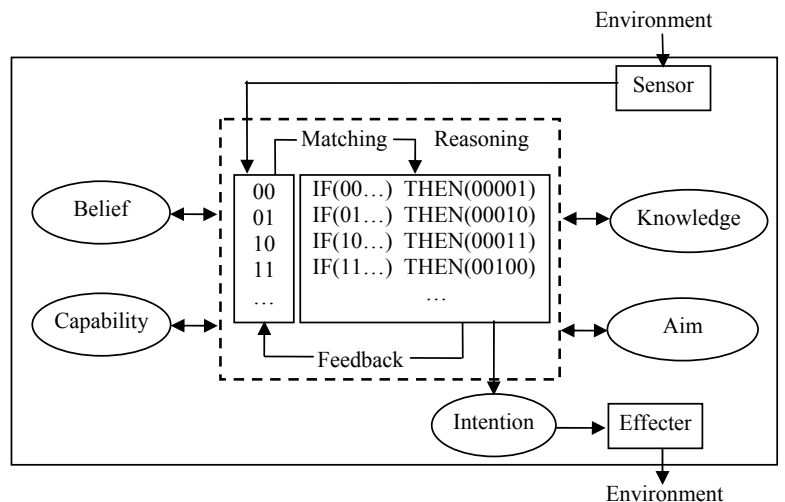

Fig. 1. Model of an entity agent.

As shown in Figure 1, an entity agent can extract rules from the data bases of belief, desire and knowledge, and 
produce intention framework by rules reasoning to create actions sequence. Furthermore, its reasoning rules can be modified according to the feedback.

Actually, the concept of intelligence means that the agent is provided with knowledge of the user's wishes and makes use of this knowledge. It also refers to the intelligent behavior emerging from the agents societies. A member in BRS executes an intelligent behavior and a sequence of data sensed by respective intelligence reconnaissance equipment. Local structures are extracted by principal component analysis, and a global map is build by integrating them. In the simulation model, a reconnaissance entity behavior can be described by a reactive rule: IF sensing condition THEN action, where a sensing condition can be directly checked on sensed values and an action can be executed by command signals. Thus, we can further design the control mechanism of a sensor reconnaissance entity agent (See Figure 2) by transforming the entity agent model in Figure 1.

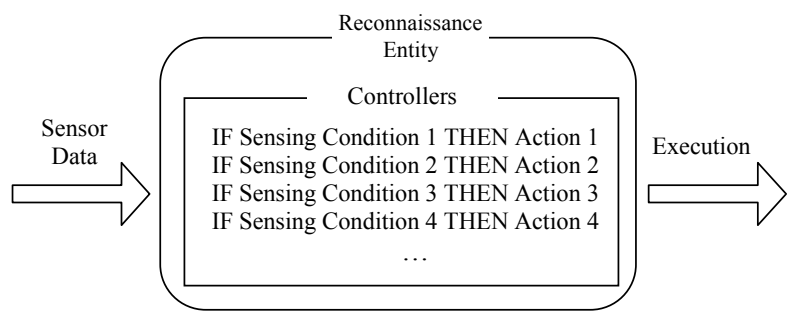

Fig. 2. Control mechanism of a sensor reconnaissance entity agent.

On information battlefield, the internal members of BRS run with autonomy and interaction. The general reconnaissance platforms (photo-reconnaissance vehicles, electronic reconnaissance vehicles, armored reconnaissance vehicles, UAVs) accept the instructions and orders from information processing vehicles, and take actions including scouting advance, alertness reconnaissance, awaiting orders. The information processing vehicles receive the intelligence from the general reconnaissance platforms, carry through information fusion and make decision. There are also a lot of cooperation, coordination and negotiation among different general reconnaissance platforms. BRS is so alike a multi-agent system in behaviors that we can set up mappings from the internal members of BRS to agents, e.g., photo-reconnaissance vehicle $\rightarrow$ photoreconnaissance vehicle agent, $\mathrm{UAV} \rightarrow \mathrm{UAV}$ agent.
In the course of the mappings, we should sort not only the function agents (multi-sensor reconnaissance platforms agents), but also the administration agents and service agents including federation manager agent, declare manager agent, federation manager agent, time manager agent, data distribute manager agent, which play the roles of demonstration control, simulation evaluation, data base, situation displaying, command practice and battlefield environment. The function agents in Red Force or Blue Force can be aggregated into the Red or Blue agents federation, and the administration agents and service agents can be aggregated into the "White" federation. In this way, we can design the basic architecture of agent-based multisensor BRS simulation as shown in Figure 3.

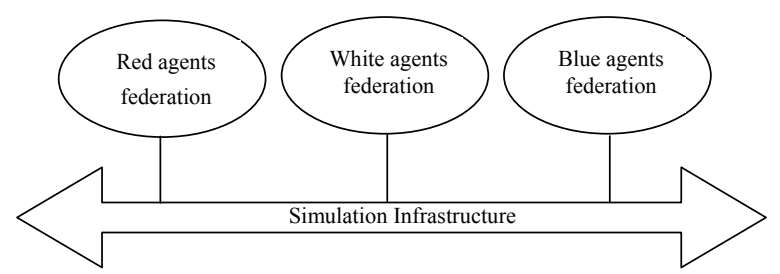

Fig. 3. Framework of agent-based multi-sensor BRS simulation.

\subsection{CNP and Collaborators}

An agent-based solution usually consists in designing and implementing some entity agents which co-operate in order to reach their internal goals. Collaboration is an interactive process among many smart mobile agents that results in varying degrees of cooperation and competition and ultimately leads to commitment. This will result in total agreement, consensus or disagreement.

CNP as well as the CNP-family protocols recognizes two roles which the involved parties may take on: an Initiator and a Participant. The Initiator is responsible for (i) initiation the collaboration, (ii) collection, evaluation and matching proposals (bids) submitted by the Participants and (iii) informing Participants about results together with finalizing the collaboration with them [25-27]. In auctions the Initiator/Participant terminology is used e.g. by FIPA and we follow it too. In contrast to the original CNP, the extended CNP model has functions of conditions constraints and confirmations for some key military tasks. 
In our case, warfare system consists of a Red armored force unit (including a command vehicle and some armored reconnaissance platforms) and a Blue army troop (including one information processing vehicle and some other reconnaissance platforms). The Contract Net initiator as a manager represents the combat command vehicle agent or information processing vehicle agent, and all other participants as contractors represent the other entity agents. The roles of manager and participants are changed once interactions relation changes.

Of course, we can also call the initiators and participants as collaborators acting as agents by CNP (See Figure 4), which are inclined towards maximizing the military profit and thus are open to collaboration.

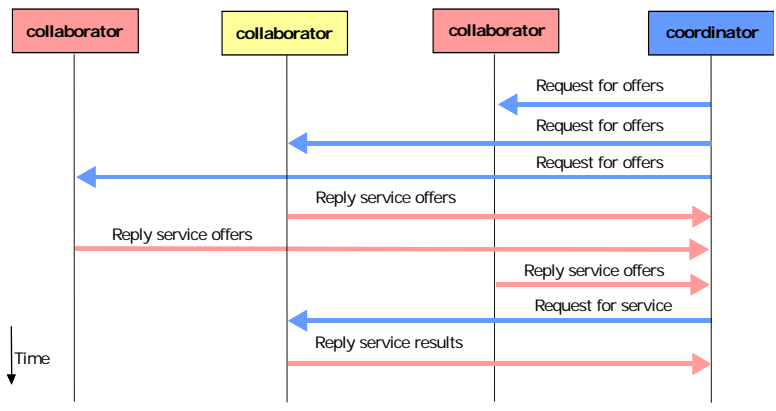

Fig. 4. Collaborators acting as agents by CNP.

\subsection{Agents Interaction Chain}

In selecting contractor in agent-based multi-sensor battlefield reconnaissance simulation, interaction is the kernel. Therefore, we should focus on interaction expression and implementation. In our model, multisensor BRS agents are connected by a chain network sharing a common knowledge and forming interaction relationship.

In order to further explore the characteristics of warfare system in agent-based simulation, we present agents interaction chain concept, since interaction process of two entity agents is essentially a hierarchical entity chain form for complex system. As shown in Figure 5, a circle represents an entity agent; $t$ means "Task Input" that drives an entity agent take its actions, expressed as a broken line arrow; while a real line arrow represents inter-agent interactions. By this rule symbol system, we can establish agents interaction chain that plays an important role in CNP mechanism and its corresponding contractor selecting approach.

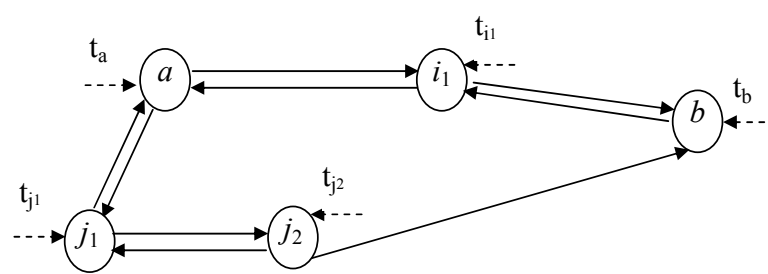

Fig. 5. Agents interaction chain.

The chain network that links the entity agents can be viewed as a collaboration graph. Thus in our model CNP can be viewed as a set of public rules that dictate the conduct of a reconnaissance entity agent with other reconnaissance entity agents to achieve a desired final outcome in sharing the knowledge and performing actions that satisfy a desired goal satisfying military functions. Therefore, agents interaction chain can be used to represent a collaboration process. This chain expresses the connectivity relationship among the reconnaissance entity agents, which reflects the contractor selecting process in CNP.

\section{Contractor Selecting Approach Based on Maximum Dynamic Integrative Trust}

\subsection{Flow Chart}

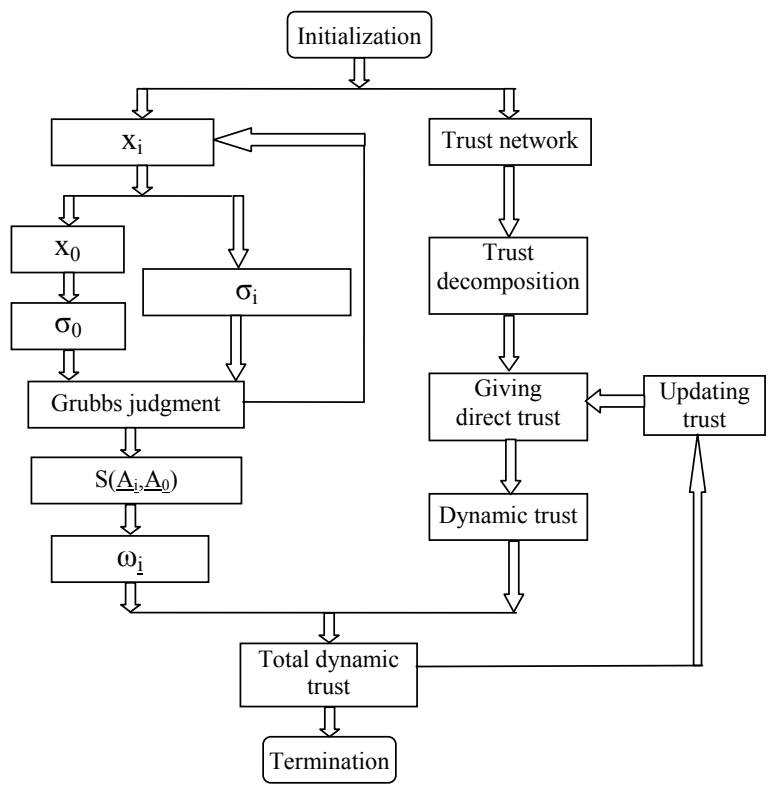

Fig. 6. Flow chart of contractor selecting approach based on maximum dynamic integrative trust. 
During multi-sensor battlefield reconnaissance agents interaction based on CNP, to select a contractor with interaction efficiency and real situation is the key. We here propose a novel approach, contractor selecting approach based on maximum dynamic integrative trust, which takes the contractor's importance weight and dynamic trust into account. The flow chart of this approach is shown in Figure 6.

In our approach, the factors of selecting contractor include the importance weight of a contractor, its performance and reliability, and its learning capability, by presenting respectively fuzzy similarity-based algorithm, agents trust network and trust modifying algorithm. Those agents fulfilling battlefield reconnaissance task in the former stage CNP interaction could be given more trust to take on a contract.

In a certain stage interaction, the selected contractor must have the maximum trust in all collaborators. Its trust can be defined as

$$
\operatorname{Trusta}_{a}(i)_{G}=\omega_{i} \operatorname{Trusta}_{a}(i) \text {. }
$$

\subsection{Computing the Importance Weights of Potential Contractors}

Multi-sensor BRS is investigated by numerous researchers. Practical applications of battlefield reconnaissance data fusion have necessarily been many areas in which the required output of an analysis may not be measured or scouted directly. This is particularly important in remote sensing such as target identification and tracking [31-32].

However, the problem of integrating results from multiple computations has been a difficulty needing to be studied by computer scientists or engineers interested in this area. Unfortunately, there are very few exceptions that can give priority to those sensor reconnaissance entities with high stability and high reliability in battlefield reconnaissance and information fusion [33-37].

Fuzzy set theory has founded successful applications in a number of fields in recent years. It will be a good tool to distinguish and evaluate the different roles of different sensor reconnaissance entities. The objective of this paper is to design a novel, fuzzy similarity-based algorithm that can synergistically combine information from sensor reconnaissance entities for reliable and accurate computing for the importance weights of a contractor in agent-based simulation by using CNP.
Battlefield reconnaissance of intelligent sensor reconnaissance platforms is usually performed in complicated circumstances. Affected synthetically by all kinds of stochastic factors, the reconnaissance values are full of uncertainty. Actually, these data which belong to a Normal Distribution [33-34] are the required samples of multi-sensor battlefield reconnaissance and information fusion.

We assume that there is a multi-sensor BRS with $n$ sensor reconnaissance entities used to carry through battlefield reconnaissance by measurement and estimation. The No. $i$ sensor reconnaissance entity is used to scout $k$ times in a short time and thereby we gets $k$ measurement values: $x_{i 1}, x_{i 2}, \ldots, x_{i k}(i=1,2,3, \ldots, n)$.

The fuzzy similarity-based algorithm is used in four stages.

In the first stage, data from multiple sensor reconnaissance entities are noted. The mean measurement value $x_{i}$, standard deviation $\sigma_{i}$ of the No. $i$ entity, and the estimated value $x_{0}$ and standard deviation $\sigma_{0}$ of the multi-sensor BRS are calculated as

$$
\begin{aligned}
& x_{i}=\frac{1}{k} \sum_{j=1}^{k} x_{i j}, \sigma_{i}=\sqrt{\frac{1}{k-1} \sum_{j=1}^{k}\left(x_{i j}-x_{i}\right)^{2}}, \\
& x_{0}=\frac{1}{n} \sum_{i=1}^{n} x_{i}, \sigma_{0}=\sqrt{\frac{1}{n-1} \sum_{i=1}^{n}\left(x_{i}-x_{0}\right)^{2}}, i=1,2, \ldots, n .
\end{aligned}
$$

In the second stage, Grubbs judgment algorithm is used to eliminate those wrong data which will reduce the precision of battlefield reconnaissance inevitably. We arrange $x_{1}, x_{2}, x_{3}, \ldots, x_{n}$ from the minimum to the maximum and give the significance $\alpha$. We define Grubbs statistic $g_{i}$ as

$$
g_{i}=\frac{x_{i}-x_{0}}{\sigma_{0}} .
$$

If this Grubbs statistic $g_{i}$ goes beyond the limit, i.e., $g_{m} \geqslant g_{0}(n, \alpha)$, then the wrongly measured datum $x_{m}$, which is the maximum or minimum, is eliminated.

In the third stage, according to fuzzy set theory, a certain sensor reconnaissance entity's measurement values and the multi-sensor BRS' estimated values are respectively expressed as normal fuzzy sets $\underline{A_{i}}$ and $\underline{A}_{\underline{0}}$. The fuzzy similarity between them is used to determine the importance weigh of a certain sensor reconnaissance entity. Thus,

$$
\mu_{\mathrm{A}_{\mathrm{i}}}(t)=\exp \left[-\left(\frac{t-x_{i}}{\sigma_{i}}\right)^{2}\right],
$$




$$
\mu_{\mathrm{A}_{0}}(t)=\exp \left[-\left(\frac{t-x_{0}}{\sigma_{0}}\right)^{2}\right] .
$$

Then, the fuzzy similarity between $\underline{A}_{\underline{i}}$ and $\underline{A}_{\underline{0}}$ can be illuminated by Figure 7 .

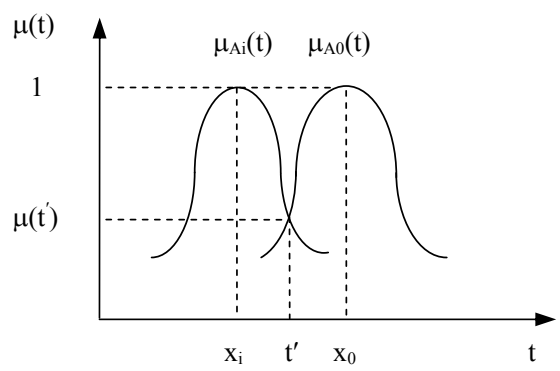

Fig. 7. Fuzzy similarity between $\underline{A_{\underline{i}}}$ and $\underline{A_{\underline{0}}}$.

According to the definition of $\underline{A}_{\underline{i}} \otimes \underline{A}_{0}, \underline{A}_{\underline{i}} \otimes \underline{A_{0}}$ is the maximum of $\underline{A}_{\underline{i}} \cap \underline{A}_{\underline{0}}$. It is actually the vertical coordinate value of the intersection of those two curves in Figure 7.

By (4) and (5), we can obtain a new equation

$$
\left(\frac{t-x_{i}}{\sigma_{i}}\right)^{2}=\left(\frac{t-x_{0}}{\sigma_{0}}\right)^{2} .
$$

Therefore,

$$
\begin{aligned}
& t^{\prime}=\frac{\sigma_{i} x_{0}+\sigma_{0} x_{i}}{\sigma_{i}+\sigma_{0}}, \\
& t^{\prime \prime}=\frac{\sigma_{0} x_{i}-\sigma_{i} x_{0}}{\sigma_{0}-\sigma_{i}} .
\end{aligned}
$$

$t^{\prime \prime}$ is beyond the bounds between $x_{i}$ and $x_{0}$, so it should be eliminated. Accordingly,

$$
\underline{A}_{i} \otimes \underline{A}_{0}=\exp \left[-\left(\frac{t^{\prime}-x_{i}}{\sigma_{i}}\right)^{2}\right] \text { or } \exp \left[-\left(\frac{t^{\prime}-x_{0}}{\sigma_{0}}\right)^{2}\right] .
$$

By associating (7) with (9), we can get the value of $\underline{A_{i}} \otimes \underline{A}_{0}$ as follows:

$$
\underline{A}_{\underline{i}} \otimes \underline{A}_{0}=\exp \left[-\left(\frac{x_{i}-x_{0}}{\sigma_{i}+\sigma_{0}}\right)^{2}\right] .
$$

According to the definition of $\underline{A}_{i} \odot \underline{A}_{0}, \underline{A}_{\underline{i}} \odot \underline{A}_{0}$ is the minimum of $\underline{A}_{i} \cup \underline{A}_{0}$. From Figure 7, we can know that the minimum is zero, i.e., $\underline{A}_{i} \odot \underline{A}_{0}=0$.

Since the similarity between fuzzy sets $\underline{A}_{i}$ and $\underline{A}_{0}$ can be defined as

$$
S\left(\underline{A_{i}}, \underline{A_{0}}\right)=\left(\underline{A_{i}} \otimes \underline{A}_{\underline{0}}\right) \wedge\left(1-\underline{A}_{\underline{i}} \odot \underline{A}_{0}\right),
$$

the similarity between fuzzy sets $\underline{A}_{i}$ and $\underline{A}_{0}$ is

$$
S\left(\underline{A_{i}}, \underline{A_{0}}\right)=\exp \left[-\left(\frac{x_{i}-x_{0}}{\sigma_{i}+\sigma_{0}}\right)^{2}\right] .
$$

$S\left(\underline{A}_{i}, \underline{A}_{0}\right)$ is larger, $\underline{A}_{i}$ is closer to $\underline{A}_{0}$. This fact shows that $S\left(\underline{A}_{i}, \underline{A}_{0}\right)$ can be viewed as the importance weigh of the No. $i$ sensor reconnaissance entity during the course of data fusion [35].

In the last stage, we calculate each entity's relative importance weigh as follows:

$$
\omega_{i}=\frac{S\left(\underline{A_{i}}, \underline{A_{0}}\right)}{\sum_{i=1}^{n} S\left(\underline{A_{i}}, \underline{A_{0}}\right)},(i=1,2, \ldots, n) .
$$

Then, we realize battlefield reconnaissance information fusion on the basis of each sensor reconnaissance entity's importance weigh calculated The final data fusion result is

$$
x=\sum_{i=1}^{n} \omega_{i} x_{i} .
$$

\subsection{Constructing Agents Trust Network}

Trust became an accredited aspect not only in social, but also in computer science [38-40]. Agent-based multisensor BRS is one of the environments where trust is indispensable and plays important roles in (i) helping determine the most reliable interaction partner (i.e., those in which the agent has the highest trust); (ii) influencing the interaction process itself (e.g., an agent's contract stance may vary according to another agent's trust level); (iii) defining the set of issues the need to be settled in the contract (i.e., the higher the trust, the more that can be left implicit in the contract).

Note that multi-sensor battlefield reconnaissance agents interaction comes into being only there is trust between collaborators. Thus, to build a trust network is an important step.

Since agents interaction chain can be viewed as a collaboration graph and reflects the contractor selecting process in CNP, we design it to be a bridge to connect trust. Thus, agents interaction chain is essentially the way of transforming real interaction relationship to a trust network where chain node agents are collaborators to be computed for trust. The method of transformation to agents trust network is to reserve the agents interaction arrows and remove other elements.

For the trust network shown as Figure 8, the trust of agent $a$ to agent $b$ can be calculate as

$$
\operatorname{Trust}_{a}(b)= \begin{cases}\operatorname{dir}_{a}(b) & (\operatorname{con} 1) \\ \operatorname{rec}_{a}\left(i_{1}\right) \times \operatorname{rec}_{i 1}\left(i_{2}\right) \times \cdots \times \operatorname{rec}_{i_{n}-1}\left(i_{n}\right) \times \operatorname{dir}_{i n}(b) & (\operatorname{con} 2) \\ 0 & (\operatorname{con} 3)\end{cases}
$$


In formula (15), con 1 means agent $a$ has a direct trust on agent $b$; con 2 means agent $a$ has a recommendation trust on agent $b$; con 3 means agent $a$ has no trust on agent $b$.

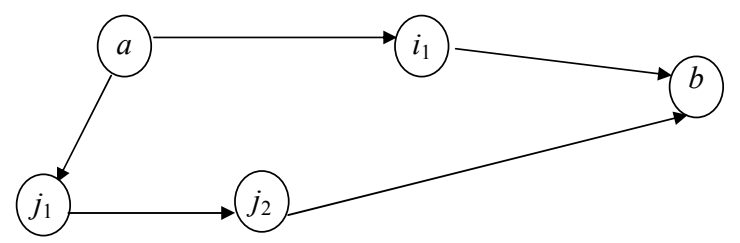

Fig. 8. Agents trust network.

\subsection{Updating the Trust of an Contractor}

The degree of trust on a contractor is updated on the basis of the degree of importance of agent in a certain interaction issue.

After an initiator (real manager) $a$ informed a contractor (real participant) $b$, the contractor performs the certain interaction issue contract through exerting its authority and capability. Thus, the initiator updates the trust on the contractor according to task performing, as

$$
\text { Trust } a(i) \leftarrow \begin{cases}\text { Trust }_{a}(i)+\xi_{s} & (\text { con } 1) \\ \text { Trust } a(i)-\xi_{f} & (\text { con } 2)\end{cases}
$$

In formula (16), con 1 means "If task is performed successfully"; con 2 means "If task is not performed"; $\xi_{s}$ and $\xi_{f}$ represent respectively the increased and decreased trust of the initiator on the contractor. Note that $\xi_{s}$ and $\xi_{f}$ are dynamic, since the trust has close relationship with the contractor's reconnaissance ability for this interaction issue, i.e., the higher the ability (the degree of importance), the more increased trust on the contractor.

Let us review the performance of two functions: $y=f_{1}(x)=\sqrt[4]{x}, y=f_{2}(x)=x^{4}(0 \leq x \leq 1)$. As shown in Figure 9, when $x$ increases, $y$ increases. However, when $x$ is closer to $0, f_{1}(x)$ increases rapidly, while $f_{2}(x)$ increases slowly; when $x$ is closer to $1, f_{1}(x)$ increases slowly, while $f_{2}(x)$ increases rapidly.
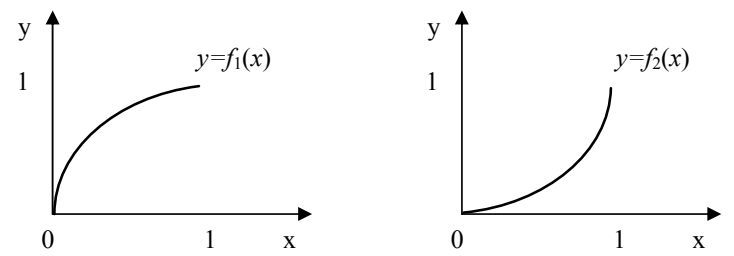

Fig. 9. Performance of functions $f_{1}(x)$ and $f_{2}(x)$.
The degree of trust belongs to $[0,1]$, so we can establish a new trust updating method as

$$
\operatorname{Trust}_{a}(i) \leftarrow \begin{cases}\min \left\{\operatorname{Trust}_{a}(i)+\sqrt[4]{\omega_{i}}, 1\right\} & (\text { con } 1) \\ \max \left\{\operatorname{Trusta}_{a}(i)-\omega_{i}^{4}, 0\right\} & (\text { con } 2)\end{cases}
$$

where con 1 and con 2 have the same meaning with formula (16) respectively.

During initialization, the trust on all latent contractors is put as 1 by the initiator. After an interaction issue, different contractors' reconnaissance results are different, i.e., their abilities and importance degrees are different, therefore, each contractor's task performing according to the contract is taken into account as trust for the next interaction issue. Thus, we can obtain a total dynamic trust to select contractor. From the view of the sensor reconnaissance entity agent, it actualizes self-learning function during this process.

\section{Case Study}

\subsection{Implementing Simulation}

The case study system that we set up can be illustrated by Figure 10. Figure 10(a) presents the agents interaction chain that expresses the relationship in dynamic and real-time military reconnaissance operations, in which $T$ and $t$ represent task inputs for Red Force agents and Blue Force agents respectively. In this case, in order to give clear expression for the difference of multi-sensor reconnaissance entities in Red Force and Blue Force, we further define the rule symbol system of agents interaction chain by supposing that the interaction operation from a entity agent in Red Force to one in Blue Force is represented as a red arrow, while the interaction operation from a entity agent in Blue Force to one in Red Force is represented as a blue arrow.

In Figure 10, different circles represent respective entity agents, as follows:

$r_{1}$ : UAV Agent 1 (Red Force);

$r_{2}$ : UAV Agent 2 (Red Force);

$r_{3}$ : Electronic Reconnaissance Vehicle Agent (Red Force);

$r_{4}$ : Photo-reconnaissance Vehicle Agent (Red Force);

$r_{5}$ : Armored Reconnaissance Vehicle Agent (Red

Force);

$r_{6}$ : Battlefield Sensor Agent 1 (Red Force);

$r_{7}$ : Battlefield Sensor Agent 2 (Red Force);

$r_{8}$ : Command Vehicle Agent (Red Force); 
$b_{1}$ : Electronic Reconnaissance Vehicle Agent (Blue Force);

$b_{2}$ : Armored Reconnaissance Vehicle Agent (Blue Force);

$b_{3}$ : Information Processing Vehicle Agent (Blue Force).

Since agents interaction chain can be used to not only actualize CNP but also establish agents trust network, we transform the above agents interaction chain in Figure 10(a) and obtain Red agent trust network and Blue agent trust network, shown as Figure 10(b) and Figure 10(c) respectively.

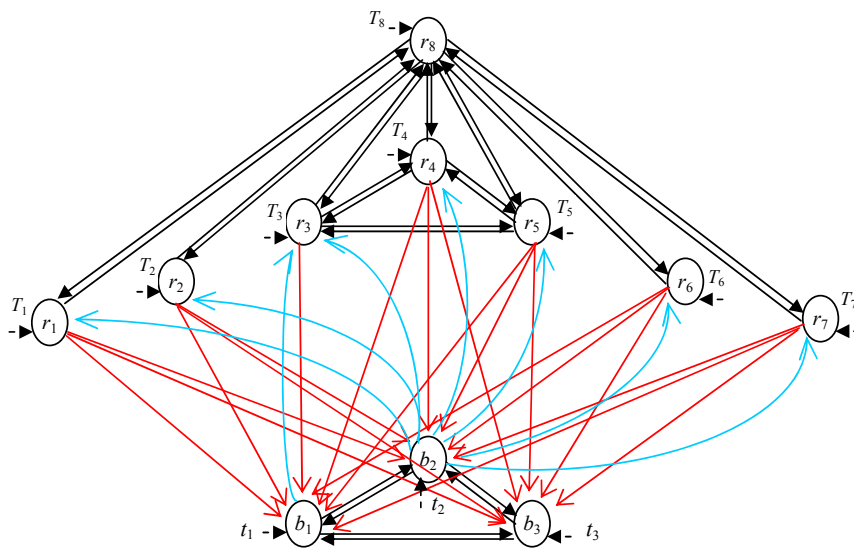

(a)

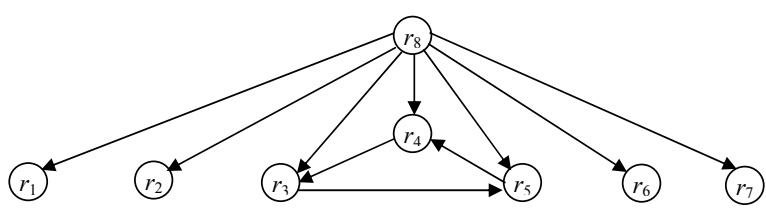

(b)

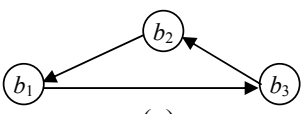

(c)

Fig. 10. Multi-sensor battlefield reconnaissance entity agents interaction chain (a), and transformation from which to Red agents trust network (b) and Blue agents trust network (c).

The interaction can be interpreted as follows.

- A call for proposals message is sent to the Participants from the Initiator following CNP.

- The Participants reply to the Initiator with the proposed intelligence reconnaissance plan times. The form of this message is either a proposal or a refusal.

- The Initiator sends accept or reject messages to Participants.
- The Participants which agree to the proposed combat plan inform the Initiator that they have completed the request to schedule warfare activities.

In this process, there are conditions constraints and iterative confirmations to ensure performing key military tasks.

The interaction is started by the information processing platform agent that acts as a manager issuing a call for proposals, e.g. scouting and measuring the No. 1 target in A region. The general reconnaissance platform agents that act as potential contractors respond with proposals, to which the information processing platform agent either rejects or accepted. Accepted proposals can be either cancelled by the information processing platform agent, or executed by a certain general reconnaissance platform agents, who later informs the information processing platform agent of success or failure of the execution. According to the real situation on information battlefield, the information processing platform agent may also re-select other proposals or issue a new call for proposals, e.g. detecting the No. 2 target in B region.

The process is a multi-agent collaboration process and a selecting contractor process according to contractor's importance weight and trust network.

The demonstration system that we set up can be illustrated by Figure 11, which presents the dynamic and real-time situation information during multi-sensor battlefield reconnaissance.

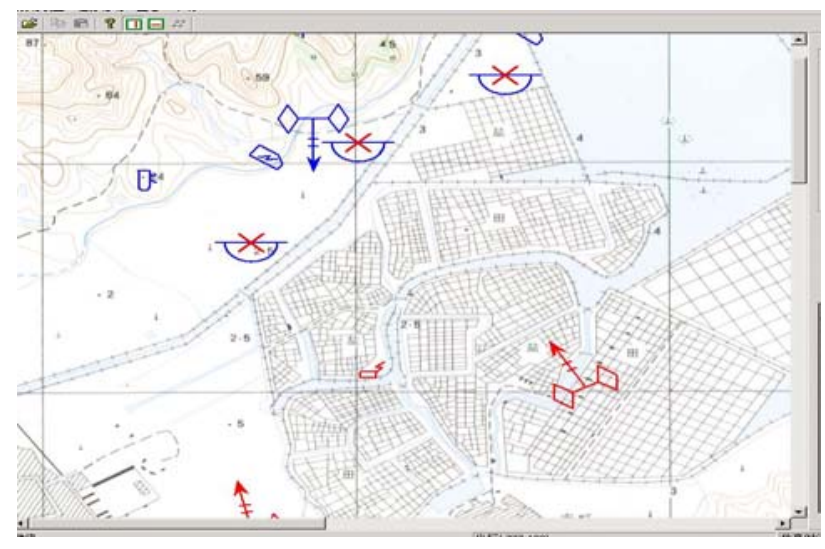

Fig.11. Two-dimension battlefield situation during military reconnaissance operations.

According to the military experiences on tactical warfare process on distributed battlefield, we can set appropriate data to the parameters for our system. When 
we run the simulation demonstration system, we can obtain some results, which are shown in Figure 12 in which $\mathrm{T}$ represents total time for fulfilling the attack battle task (minute), E represents attack efficiency ( $\mathrm{min} /$ target) and $\mathrm{R}$ represents rate of destroyed force (\%). In Scenario A, the Red armored force unit takes a transverse deployment. Column and triangular deployment are taken respectively in Scenario B and Scenario C. Thus by these simulation results one can find that Scenario $\mathrm{C}$ is the most effective attack battle plan for the Red armored force unit while Scenario B is the worst one.

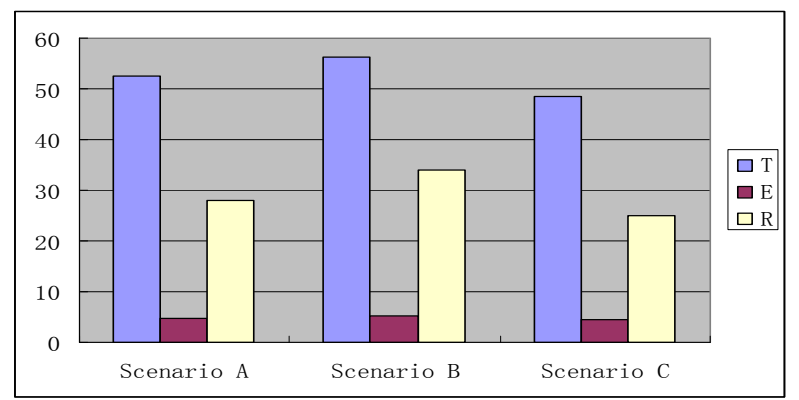

Fig.12. Contrastive results of three scenarios.

\subsection{Contractor Selecting Results and Checking Model}

In this case, we measure and estimate some targets or obstacles in the land battlefield environment multisensor reconnaissance entity agents fall across. The obtained reconnaissance measurement values are recorded in Table 1.

Table 1. Measurement values of multi-sensor reconnaissance entity agents.

\begin{tabular}{cccccc}
\hline Agent & 1 & 2 & 3 & 4 & 5 \\
\hline No.1 Measurement value & 43.1 & 41.5 & 44.6 & 39.7 & 48.1 \\
No.2 Measurement value & 43.4 & 41.1 & 44.3 & 39.9 & 47.4 \\
No.3 Measurement value & 43.8 & 41.4 & 45.1 & 40.2 & 47.2 \\
$\ldots$ & $\ldots$ & $\ldots$ & $\ldots$ & $\ldots$ & $\ldots$ \\
\hline
\end{tabular}

By computation according to formula (1), we can attain the mean measurement values of each sensor reconnaissance entity agent: $x_{1}=43.5, x_{2}=41.4, x_{3}=44.8$, $x_{4}=39.8, x_{5}=47.6$; standard deviation $\sigma_{1}=0.316, \sigma_{2}=0.216$, $\sigma_{3}=0.424, \sigma_{4}=0.337, \sigma_{5}=0.392$.

Each sensor reconnaissance entity agent's fuzzy similarity and relative importance weigh can be shown in Table 2.
Table 2. Each sensor reconnaissance entity agent's fuzzy similarity and relative importance weigh.

\begin{tabular}{lccccc}
\hline \multicolumn{1}{c}{ Agent } & 1 & 2 & 3 & 4 & 5 \\
\hline $\begin{array}{l}\text { Fuzzy similarity } \\
\begin{array}{l}\text { Relative importance } \\
\text { weigh }\end{array}\end{array}$ & 0.976 & 0.678 & 0.852 & 0.313 & 0.224 \\
\hline
\end{tabular}

According to our data fusion algorithm, the final data fusion result can be also attained:

$$
x=\sum_{i=1}^{5} \omega_{i} x_{i}=43.314
$$

Table 2 shows that the measuring stability and reliability of the 1st sensor reconnaissance entity agent is the maximum, the 3rd one takes second place, and the 5 th one is the minimum. In fact, the 1 st sensor reconnaissance entity agent's mean measurement value $x_{1}=43.5$, which is the nearest data to the final reconnaissance data fusion result 43.314. On the contrary, the 5th agent's mean measurement value $x_{5}$ is the furthest data to the final reconnaissance result. Experimental result indicates that the algorithm can give priority to the high-stability and high-reliability sensor reconnaissance entity agents.

In fact, we perform an actual reconnaissance measurement and find that this distance is 43.348 . Therefore, through the fuzzy similarity-based algorithm the error is only 0.034 , and the relative error is only $0.0784 \%$. Through those algorithms in [33] and [34], however, the errors are respectively 0.054 and 0.042 . Accordingly, the relative errors are respectively $0.125 \%$ and $0.0969 \%$ (See Figure 13). It shows that the precision of the multi-sensor reconnaissance data fusion in battlefield reconnaissance can be improved by using our algorithm.

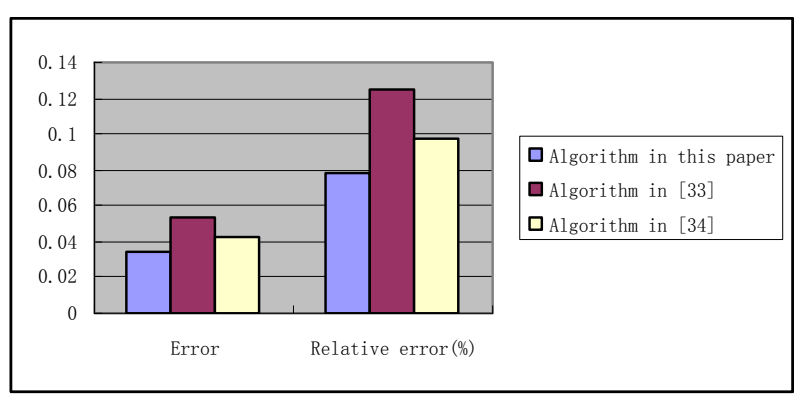

Fig. 13. Contrastive results of three algorithms.

The experiments are performed again and again. The results are well accordant with the real data. 
Based on the computation of the importance weighs of contractors, if this interaction is the first contract issue, the trusts of the initiator (information processing platform agent) on all contractors (general reconnaissance platform agents) are 1. Thus, according to formula (19), the 1st general reconnaissance platform agent is selected as the current contractor since the trust of the initiator on it is the maximum.

$$
\text { Trust }{ }_{a}(1)_{G}=\max \left\{\omega_{i} \text { Trust }_{a}(i) \mid i=1,2, \ldots, n\right\}=0.321
$$

After the 1st general reconnaissance platform agent performed the current interaction issue contract, the trust of the initiator on it is updated as

$$
\text { Trust } \begin{aligned}
a(1)_{n e x t} & =\min \left\{\text { Trust } a(1)+\sqrt[4]{\omega_{1}}, 1\right\} \\
& =\min \{0.321+0.753,1\}=1
\end{aligned}
$$

If it fails to perform this contract task, the trust of the initiator on it is updated as

$$
\begin{aligned}
\operatorname{Trusta}_{a}(1)_{\text {next }} & =\max \left\{\operatorname{Trust}_{a}(1)-\omega_{1}^{4}, 0\right\} \\
& =\max \{0.321-0.011,0\}=0.310
\end{aligned}
$$

Thus, we can obtain this fact that the selected contractor in the first interaction issue, by its relative best performance in contract task, can win a better station in the next interaction issue. Even it didn't achieve its established objective in contract, it still holds a relative high trust 0.310 . We further analyze the process and can find that the method is applicable to other interaction issues in latter stages. The fact fitly reflects the rationality of our model.

By implementing verification, validation, and accreditation, the results that we obtained from agentbased multi-sensor battlefield reconnaissance simulation by CNP is accordant to real military situation. The fact proves that our model is feasible and effectual.

\section{Conclusion}

Agent-based simulation has become a popular simulation analysis tool and has been used to examine complex warfare system like BRS. We take agent-based multi-sensor battlefield reconnaissance simulation as research object. One of kernels in agent-based simulation is agents interaction and its protocol. In CNP mechanism, how to select a reasonable contractor is a challenging problem. In this paper, we propose a novel approach based on maximum dynamic integrative trust for it. After analyzing agent and agent-based simulation framework, CNP and collaborators, and agents interaction chain, we present the flow chart and basic steps of our contractor selecting approach and validate its feasibility and efficiency by case study. In contrast with other current approaches, our approach has some advantages: (1) taking the importance weights of potential contractors into account during selecting contractor; (2) presenting a method on building agents interaction chain and transforming it to corresponding trust network that embodies the process of selecting contractor; (3) putting forward a means of modifying dynamically the trust of an contractor. Thus, the approach is applicable in describing the agent-based simulation model in which military entities collaborate to carry out reconnaissance task with high efficiency.

\section{Acknowledgements}

This research was supported by the National Natural Science Foundation of China (Grant No. 70901075), Military Science Projects for Graduate Supervisors (Grant No. 2010JY0636-366, No. 2011JY001-015) and Innovation Foundation of AAFE (Grant No. 2011CJ057).

\section{References}

1. M. Wooldridge and N. R. Jennings, The cooperative problem-solving process, Journal of Logic and Computation, 9(4) (1999) 563-592.

2. V. Lesser, Autonomous Agents and Multi-Agent Systems (Kluwer, Boston, 1998).

3. Anita Raja and Victor Lesser, A Framework for metalevel control in multi-agent systems, Autonomous Agents and Multi-Agents Systems, 15(2) (2007) 147-196.

4. Kota, N. M. Gibbins, N. R. Jennings, Self-organizing agent organizations, in Pro. Int. Conf. Autonomous Agents and Multi-Agent Systems (2009), pp. 797-804.

5. Zhongzhi Shi, Intelligent Agents and Their Applications (Science Press, Beijing, 2001).

6. Zhongzhi Shi and Nanning Zheng, Progress and challenge of artificial intelligence, Journal of Computer Science and Technology, 21(5) (2006) 810-822.

7. Linxuan Zhang, Tianyuan Xiao, $\mathrm{Ce}$ Liang, Implementation of an ASP-oriented distributed collaborative design system and its applications in pervasive computing environment, in Pro. 2nd Int. Conf. Pervasive Computing and Applications (2007), pp. 133138.

8. Arthur Huang, David Levinson, Why retailers cluster: an agent model of location choice on supply chains. Environment and Planning B: Planning and Design, 38(2011) 82-94.

9. Shoham, Yoav, Kevin Leyton-Brown, Multi-agent Systems: Algorithmic, Game-Theoretic, and Logical Foundations (Cambridge University Press, 2009). 
10. Krzysztof Juszczyszyn, Grzegorz Kolaczek, Attack pattern analysis framework for a multiagent intrusion detection system, International Journal of Computational Intelligence Systems, 1(3) (2008) 215-224.

11. A. Zunino, M.Campo, Chronos: A Multi-agent system for distributed automatic meeting scheduling, Expert Systems with Applications, 36(3) (2009) 7011-7018.

12. Jorge E. Hernandez, Josefa Mula, Raul Poler, Juan Pavon, A multiagent negotiation based model to support the collaborative supply chain planning process, Studies in Informatics and Control, 20(1) (2011) pp. 43-54.

13. Dong Menggao, Mao XinJun, Chang ZhiMing, Wang Ji, Qi Zhichang, Running mechanism and strategy description language SADL for self-adaptive MAS, Journal of Software, 22(4) (2011) 609-624.

14. Franziska Klügl, A validation methodology for agentbased simulations, in Pro. ACM Symposium on Applied Computing Table of Contents (2008), pp. 39-43.

15. R. Gore and P. F. Reynolds Jr, INSIGHT: understanding unexpected behaviors in agent-based simulations, Journal of Simulation, 4(3) (2010) 170-180.

16. Xiong Li and Zhiming Dong, Platform-level distributed warfare model-based on multi-agent system framework, Defence Science Journal, 62(3) (2012) 180-186.

17. Guoyin Jiang, Bin Hu, Youtian Wang, Agent-based simulation of competitive and collaborative mechanisms for mobile service chains, Information Sciences, 180(2) (2010) 225-240.

18. Xiong $\mathrm{Li}$, Zongchang $\mathrm{Xu}$, Zhiming Dong and Yiwei Zhang, Formal specification of agent-oriented multiple sensors system organization based on Object-Z, Information-An International Journal, 15(8) (2012), pp. 3585-3594.

19. Ibrahim Cil, Murat Mala, A multi-agent architecture for modeling and simulation of small military unit combat in asymmetric warfare, Expert Systems with Applications, 37(2) (2010) 1331-1343.

20. Xiong Li, Gaotian Pan, Zhiming Dong, Dianbo Cui and Hongwei An, Designing of multi-agent-based of complex Warfare system simulation model, Dynamics of Continuous, Discrete and Impulsive Systems, Series A: Mathematical Analysis, 13(S3) (2006) 953-959.

21. Bikramjit B., Ahmed A., Landon K., Layered intelligence for agent-based crowd simulation, Simulation, 85(10) (2009) 621-633.

22. Guoyin Jiang, Bin Hu, and Youtian Wang, Agent-based simulation approach to understanding the interaction between employee behavior and dynamic tasks, Simulation, 87(5) (2011) 407-422.

23. Gyoo Gun Lim, Kun Chang Lee, Won Jun Seo and Dae Chul Lee, Multi-agent based simulation for evaluation of mobile business models, Information-An International Journal, 14(9) (2011), pp. 3063-3080.

24. FIPA: Foundation for Intelligent Physical Agents, (2011) http://www.fipa.org (accessed 08.07.11)

25. K. Junichi, H. Tomoki, H. Shinji, T. Tanabe, T. Funabashi, H. Hirata, Multi-agent-based autonomous power distribution network restoration using contract net protocol, Electrical Engineering in Japan, 166(4) (2009) 56-63.

26. R. G. Smith, The contract net protocol: high level communication and control in distributed problem solver, IEEE Transactions on Computers, 29(12) (1980) 11041113.

27. David Jégou, Dae-Won Kim, Pierre Baptiste, Kwang H. Lee, A contract net based intelligent agent system for solving the reactive hoist scheduling problem, Expert Systems with Applications, 30(2) (2006) 156-167.

28. E. Durfee, Coordination of Distributed Problem Solvers (Kluwer, Boston, 1988).

29. S. Paurobally, J. Cunningham, N. R. Jennings, Verifying the contract net protocol: a case study in interaction protocol and agent communication language semantics, in Pro. 2nd Int. Conf. Logic and Communication in Multi-Agent Systems (2004), pp. 98-117.

30. N. Haque, N. R. Jennings, L. Moreau, Resource allocation in communication networks using marketbased agents, in Proc. 24th Int. Conf. Innovative Techniques and Applications of AI (2004), pp. 187-200.

31. Z. Korona and M. Kokar, Model-based fusion for multisensor target recognition, in Proc. SPIE, Vol. 2755 (1996), pp. 178-189.

32. A. Gad and M. Farooq. Data fusion architecture for maritime surveillance, in Proc. 5th Int. Conf. Information Fusion (2000), pp. 448-455.

33. Xiang Xinjian, A method to sensor data fusion based on fuzzy and statistics integration, Chinese Journal of Sensor Technology, 23(2) (2004) 197-199.

34. Wei Suiyi and Liu Guixiong, Novel arithmetic of similar multi-sensor data fusion, Journal of Transducer Technology, 23(8) (2004) 61-65.

35. Chen Zhining and Chen Lixin, Fuzzy Set Theory and Its Applications (Artillery Academy, Heifei, 2000).

36. Q. Gan and C. J. Harris, Comparison of two measurement fusion methods for Kalman-filter-based multi-sensor data fusion, IEEE Transactions on Aerospace and Electronic Systems, 37(1) (2001) 273-279.

37. G. Girija, R. A. Raj, J. R. Raol, Tracking filter and multisensor data fusion, Sadhana, 25(2) (2000) 159-167.

38. F. E. Walter, S. Battiston, F. Schweitzer, Personalized and Dynamic Trust in Social Networks. in Pro. 3rd ACM Conf. Recommender Systems (2009), pp. 197-204.

39. F. Skopik, D. Schall, S. Dustdar, Modeling and mining of dynamic trust in complex service-oriented systems, Information Systems, 35(7) (2010), 735-757.

40. P. Bedi, A. K. Sinha, S. Agarwal, A. Awasthi, G. Prasad, D. Saini, Influence of terrain on modern tactical combat: trust-based recommender system, Defence Science Journal, 60(4) (2010) 405-411. 